\title{
AUX ORIGINES DE LA PENSÉE POLITIQUE AMÉRICAINE
}

Dick Howard*

SÍNTESE - O artigo busca refletir sobre as eleições de 2004 nos EUA, colocando-as em seu contexto histórico e filosófico, de forma a revisitar as origens revolucionárias do pensamento político americano em uma análise fenomenológica que desvela em que sentido a democracia pode ser dita radical.

PALAVRAS-CHAVE - Democracia. Eleições. Pensamento politico. Revolução americana.
ABSTRACT - The article tries to reflect on the 2004 US elections by putting them in historical and philosophical context, so as to recast the revolutionary origins of the American political thought in a phenomenological analysis that unveils in which sense democracy may be said to be radical.

KEY WORDS - American revolution. Democracy. Elections. Political thought.

\section{Introduction: Quel avenir pour la démocratie américaine?}

Faut-il souligner les 3,5 millions de voix qui ont porté George W. Bush à la présidence, ou les $51 \%$ des électeurs que ce chiffre représente? Le vainqueur comme le vaincu appellent à l'unité après une bataille âpre qui rappelle à l'historien l'élection de 1800 qui opposa l' "athée francophile" Jefferson au "monarchiste anglophile" John Adams. De la division sortit pourtant l'affirmation des institutions qui encadrent la société démocratique. Qu'en est-il aujourd'hui?

On dit que George Bush vient de gagner la légitimité qui lui faisait défaut depuis qu'un arrêt de la Cour suprême a consacré, en décembre 2000, la légalité d'une victoire électorale malgré le fait que son opposant avait obtenu la « majorité des voix populaires ». Beaucoup s'étonnèrent à l'époque que le public ait accepté cette élection judiciaire sans protestations. Cela s'explique par le fait que M. Bush s'était alors présenté comme un homme politique conciliateur, un "conservateur compassionnel" qui, du fait même de son absence de mandat, devrait chercher l'appui public le plus large, donc gouverner au centre.

Or, la politique radicale du nouveau président s'est manifestée très tôt, bien avant le choc du 11 septembre qu'il a interprété comme un moment providentiel,

Doutor. Professor, State University of New York at Stony Brook, USA.

\begin{tabular}{|l|l|l|l|l|l|}
\hline VERITAS & Porto Alegre & v. 50 & n. 1 & Março 2005 & p. 101-125 \\
\hline
\end{tabular}


comme la confirmation de sa mission politique et le défi auquel devait s'affronter sa volonté morale. Cette radicalité du gouvernement Bush explique en grande partie le choix de John Kerry comme candidat démocrate dont la plateforme se résumait (malgré lui) à être l'anti-Bush. Elle fut marquée non seulement par une réduction des impôts au bénéfice des plus fortunés mais aussi par le rejet du traité de Kyoto ainsi que l'accord sur les missiles anti-balistiques (afin de poursuivre la mise en place du système anti-missiles star wars). L'expression symbolique de cette politique fut le refus du vice-président Cheney de rendre public les noms des conseillers (lobbyistes et industriels) qui lui avait inspiré sa politique énergétique. Ce style quasi-conspirationnel s'accentuera après le 11 septembre.

À la lumière de cette lecture du premier mandat - légal mais non légitime on pourrait craindre que la victoire populaire accentuera la poussée radicale du gouvernement Bush. Le président étant fort d'un Congrès où la majorité républicaine s'est accrue dans les deux chambres, et de la possibilité de nommer plusieurs juges à la Cour suprême, ceux qui soutenaient John Kerry - en tant qu'antiBush - craignent le pire. Le scénario " optimiste » suppose que Bush pourrait être victime de sa propre hubris : faute de savoir reconnaitre ses propres limites, il devrait finir par s'autodétruire. Un tel "optimisme " reflète un pessimisme qui n'ose pas dire son nom.

Raisonner ainsi, c'est oublier qu'à l'opposé du candidat de l'an 2000, le président sortant s'est fait réélire sur une plateforme qui n'en est pas une. Son projet politique, c'est sa personne qui l'incarne. Dénonçant son opposant comme une "girouette" opportuniste incapable de faire face aux velléités de l'opinion publique (globale? française?), le présidant insistait sur son "caractère" personnel et sa volonté morale. À ce volontarisme du moraliste correspondait une politique étrangère fondée sur la logique sportive selon laquelle la meilleure défense serait une bonne attaque. Ainsi le réalisme traditionnel du multilatéralisme selon lequel la guerre ne se justifie que comme une réaction pré-emptive commune à une agression effective est remplacé par une politique préventive dont le présupposé implicite est celui d'un monde hobbesien (homo homini lupus) où celui qui frappe le premier sortira vainqueur.

En d'autres termes, George Bush s'est présenté comme l'incarnation d'une politique de la volonté. Or, une telle politique moralisatrice peut s'accommoder de n'importe quel contenu. C'est cela qui explique que, malgré l'effet mobilisateur fourni par les onze referendums sur le mariage homosexuel, les majorités renforcées dans les deux chambres furent élues - souvent de peu - sur des questions de portée strictement locale.

Une telle politique de la volonté met en question les acquis fondamentaux de la démocratie. Cette dernière est fondée sur l'égalité de principe qui fait que chacun à droit à sa différence, que tous les intérêts doivent pouvoir s'exprimer afin que leur confrontation produise un jugement délibéré sur ce que l'on reconnait comme le bien commun.

L'attrait manifeste de cette politique de la volonté s'explique d'abord par la crainte existentielle née des attentats du 11 septembre. Face à la menace, l'unité 
s'impose, une nation divisée est incapable de se défendre comme une volonté divisée ne peut pas vouloir. ${ }^{1}$ G. Bush n'a d'ailleurs pas cessé de rappeler le danger terroriste et de souligner les vertus de sa politique préventive. D'après un sondage à la sortie des urnes 54\% des électeurs identifient la guerre en Iraq et la lutte contre le terrorisme; cela explique que ni l'absence d'armes de destruction massive, ni les crimes d'Abu Graib, ni non plus la disparition de 370 tonnes d'explosifs en Iraq n'ont détourné les électeurs de Bush.

La politique de la volonté menace également la démocratie par son moralisme. On trouve là encore dans l'opinion publique les traces du 11 septembre. De même que le moraliste ne met jamais en question ses bonnes intentions, de même les Américains ne comprennent pas qu' "on" leur en veuille à ce point. Incapables de regarder en face leurs propres actions, les moralistes ne peuvent pas prendre au sérieux le jugement des autres; ils deviennent autistes. Le résultat concret en est une incapacité des Américains à faire de leur puissance réelle un pouvoir légitime qui n'aurait pas besoin de la force des armes pour imposer leur vision démocratique.

Avant de céder à la tentation de déplorer l'utilisation cynique des craintes suscitées par le 11 septembre - et surtout, avant d'admettre que la bonne mobilisation citoyenne, grâce à laquelle le taux de participation a dépassé les attentes pour retrouver celui de l'élection de John F. Kennedy en 1960, comportait aussi une nette augmentation de la mobilisation des "fondamentalistes" antihomosexuels et anti-avortement - il faut reconnaître que cette politique de la volonté si bien mise en œuvre par les conseillers de Georges Bush est inhérente à la démocratie elle-même. La démocratie est un jeu dangereux. Une phrase de Tocqueville résume les difficultés qui lui sont propre: "Je n'aime pas la démocratie pour ce qu'elle est, mais pour ce qu'elle fait faire." C'est la société civile qui constitue l'essence de la démocratie. Mais celle-ci n'est pas une donnée naturelle ; elle ne peut exister qu'à partir d'institutions politiques qui l'encadrent et la sollicitent quand elle s'inquiète des divisions et conflits qui se trouvent à la racine de son activité sans cesse renouvelée. Telle est la racine de la politique de la volonté - qui n'est pas une simple réaction au 11 septembre : pour dépasser la division pourtant constitutive de la société civile démocratique, l'on cherche une unité transcendante - soit morale, soit nationale (ou patriotique).

Revenons maintenant à la campagne politique que nous venons de vivre - et rappelons qu'elle fut aussi hargneuse et centrée sur des questions fondamentales que celle de 1800. Elle peut se réduire à l'opposition entre une politique de la volonté et une politique du jugement. Souvenons-nous du second débat. À la question du droit à l'avortement (du "choix" de la femme, disent les démocrates), Kerry a répondu par une analyse de situations particulières qui nuancent le jugement à y porter, alors que pour Bush la réponse tenait en une phrase. Or, de l'avis

En termes durkheimiens, la solidarité ainsi créée ne serait que "mécanique" alors qu'une solidarité "organique" devrait se fonder sur ce que j'appelle un jugement délibératif. 
presque général, Kerry est sorti des trois débats comme le candidat. Et pourtant, entre les débats et l'élection, il a fait piètre figure. Pourquoi?

Une politique de la volonté mobilise, une politique du jugement délibère, se critique, fait de ses hésitations une vertu. ${ }^{2}$ Comment Kerry, aurait-il pu faire pour conserver son avantage dans le régime de démocratie médiatique que l'on connait aujourd'hui ?

Mon propos n'est pas de faire revivre la campagne maintenant terminée ; je m'appuie au contraire sur l'expérience historique pour réfléchir sur le présent. Si tant est que la comparaison avec l'élection de 1800 tient la route, il faut se demander quelles nouvelles institutions politiques et citoyennes pourraient sortir de cette élection si contestée. Quel sera, par exemple, l'avenir du mouvement citoyen - aussi bien chez les libéraux démocrates que chez les moralistes républicains - dont l'importance frappe tous les observateurs? Malgré l'absence presque total de soutien au candidat alternatif Ralph Nader, la mobilisation de la société civile (hors des partis) mérite d'être soulignée. Peut-on imaginer, par exemple, que cette mobilisation évolue et prenne la forme d'ONG domestiques? Une nouvelle politique des droits de l'homme, qui rappelle celle qui avait vaincu feu le totalitarisme, pourrait se profiler à l'horizon.

Vouloir conclure sur une note positive serait trahir la logique du jugement. Au lendemain de l'élection, lorsqu'on extrapole les mesures qui ont marqué le premier mandat Bush (déficit budgétaire et commercial, réduction des impôts des riches, politique écologique, secret administratif, etc.), on est en droit d'être pessimiste. Pourtant, la mise en perspective aussi bien historique (1800) que philosophique (quid l'avenir de la démocratie ?) en appelle au jugement. Celui-ci, faut-il rappeler, constitue également une manière de faire de la politique. Exerçons ce droit fondamental et constitutif de la démocratie.

\section{Aux origines de la révolution américaine}

Comme sa cousine française, la révolution américaine se prête à des relectures qui sont autant de symptômes de l'actualité contemporaine. En voici encore une, qui de surcroît s'offre au public français. Elle propose une double démarche. Il s'agit d'abord de comprendre l'origine d'une pensée politique qui réussit à instaurer une république démocratique, ce qui devrait ensuite permettre au lecteur d'interroger l'évolution et la forme actuelle prise par celle-ci. Plus spécifiquement, il s'agit d'analyser la mise en place pratique et théorique d'une politique du jugement, mais aussi de comprendre les dérapages possibles d'une démocratie qui prétend exprimer la volonté populaire. Montrer ainsi le travail créateur du jugement en politique devrait permettre aux Européens de dépasser un antiaméricanisme simpliste pour mieux comprendre, et donc mieux critiquer, la politique actuelle des États-Unis (qu'elle soit dirigée par l'un ou l'autre des partis politi-

On croirait entendre ici les paroles de Marx à propos de la Commune de Paris! Mais c'est une autre histoire - qu'il faudrait quand même rappeler aux déçus de la gauche. 
ques qui prétendent incarner cette volonté populaire). Regarder la mise en forme et la mise en oeuvre du jugement politique, devrait permettre au lecteur d'affiner sa propre aptitude de juger son actualité.

Si le rêve de la démocratie remplace aujourd'hui celui de la révolution, peuton en conclure que l'étude de la révolution américaine devrait remplacer celle de sa cousine française, titulaire depuis longtemps du titre de modèle politique? Que la thèse d'une "exception américaine " ai été inventée pendant les années 1930 par des dissidents communistes pour se libérer de l'emprise du dogmatisme historiciste imposé par Moscou, n'incite pas à lui accorder une telle priorité. S'il s'agissait vraiment d'une exception, il faudrait mettre en doute l'existence d'une règle qui en justifierait le transfert et l'application dans un autre contexte et dans des conditions différentes, car comme sa cadette, elle deviendrait un leurre idéologique du même genre que l'histoire romaine dont Marx dénonçait l'effet nocif sur les révolutionnaires français.

Inutile donc d'insister: mon propos ne veut ni défendre les institutions républicaines et démocratiques inventées et mises en œuvre à partir de la révolution américaine, ni en tirer des leçons que l'on plaquerait sur les problèmes actuels. Disons plutôt que l'expérience américaine est exemplaire, au sens kantien du terme. Il s'agit d'une expérience inédite, rebelle à toute interprétation à partir de schémas hérités, et condamnée à une recherche toujours renouvelée de comprendre l'histoire en train de se faire. C'est pourquoi elle sollicite le jugement politique ${ }^{3}$.

La forme républicaine finalement adoptée par la démocratie américaine n'était pas celle qui inspirait sa lutte pour l'indépendance, ni non plus celle qui fut tout d'abord mise en œuvre par le jeune pays lorsqu'il est devenu souverain. Il fallait inventer une manière de représenter cette souveraineté sans prétendre l'incarner et donc la fixer une fois pour toutes. Comment instituer une république qui peut à la fois encadrer et encourager une société démocratique ouverte alors que l'activité incessante de la société libre en menace la stabilitét? Il fallait non seulement produire une théorie politique ; il fallait aussi la mettre en œuvre, ce qui n'allait pas de soi. On cite souvent le mot du vieux Benjamin Franklin, décrivant

L'opposition entre une histoire exceptionnelle et une histoire exemplaire est proposée par John Patrick Diggins, On Hallowed Ground. Abraham Lincoln and the Foundations of American History (New Haven, Yale University Press, 2000), pp. 105 sq. C'est moi qui en propose une lecture kantienne - qui, bien sûr, n'était pas celle des acteurs eux-mêmes.

4 L'idée que les institutions républicaines encadrent et encouragent la société démocratique distingue la démocratie républicaine à l'américaine de la république démocratique visée implicitement par la révolution française et réalisée (mal) par la révolution russe de 1917 et ses imitateurs. Malgré les différences qui n'ont pas besoin d'être soulignées, Charles Kupchan n'hésite pas à mettre en rapport le travail institutionnel de la révolution américaine et celui qui reste à faire pour créer une vraie unité européenne. Il s'agit parfois simplement d'analogies suggestives, mais certaines thèses théoriques vont plus loin et reprennent des arguments tirés ici de l'expérience américaine, comme celui d'une auto-limitation de la souveraineté qui va de pair avec l'encadrement et l'encouragement d'une société démocratique. Cf. Charles A. Kupchan, The End of the American Era. U.S. Foreign Policy and the Geopolitics of the Twenty-First Century (New York, Knopf, 2002), par exemple les pages 133sqq., 146 et surtout 248sqq. 
les résultats de la réunion constitutionnelle de Philadelphie: "Une république, si vous êtes capables de la maintenir ${ }^{5}$."

De quelle manière doit-on raconter cette histoire originaire dont les avatars restent toujours présents alors que nos contemporains n'en sont pas toujours conscients? Comme toute origine, elle ne se comprend qu'à partir de ses diverses et multiples réalisations. Le problème est d'autant plus difficile que les acteurs eux-mêmes n'étaient pas pleinement conscients de la portée de leurs innovations. Il faut évidemment suivre la chronologie, mais celle-ci n'acquiert une voix qu'au travers des représentations qu'en ont données les participants. Il s'agit donc de décrire une pratique à partir de ses présupposés théoriques pour ensuite regarder comment le décalage entre la théorie et sa mise en cauvre conduit les participants à repenser la première. Malgré mon refus de l'anachronisme, l'expérience américaine représente de façon exemplaire ce qu'on peut décrire, en reprenant les catégories de Claude Lefort, comme une mise en scène, en forme et en sens de ce qu'est le jugement politique ${ }^{6}$. Avant d'entrer plus dans la chair de l'histoire, il faut expliquer la portée de ce concept qui n'apparait ni dans le vocabulaire des acteurs ni dans ma "reconstruction" de leurs actions. Et pourtant, on le verra, il est omniprésent dans ce récit, auquel il donne sa structure logique.

$\mathrm{Au}$ niveau le plus simple, on peut dire que l'article lui-même est l'articulation d'une forme du jugement politique. Chaque partie présente trois étapes au travers desquelles l'expérience se déroule. Il s'agit tout d'abord d'interpréter le vécu immédiat des gens, ce qui, bien sûr, repose sur l' expérience préalable des acteurs. Il ne s'agit donc pas une positivité neutre que l'analyse pourrait survoler. Des lignes de force, des tensions ainsi que des interventions humaines font vibrer ce vécu; mis en branle, il faudra le reprendre et lui donner des formes institutionnelles qui le fixent et en permettent la conceptualisation. Ce deuxième moment, le conçu, représente une généralisation qui présente l'avantage de comprendre (au sens littéral et figuratif) la particularité du vécu. Mais il comporte aussi un risque, car il tend à absorber le vécu, à l'étrangler en le coupant de sa racine vivante. C'est alors qu'entre en jeu le troisième moment, le réfléchi. Celui-ci devra trouver une sorte de médiation qui permette aux deux premiers moments de conserver leur originalité. Par cette étape de réflexion, ils se trouvent maintenant dans un rapport de complémentarité l'un par rapport à l'autre. Une telle médiation ne propose pas une synthèse du genre hégélien qui dissout et recompose sans restes les matériaux qui entrent en jeu. Il s'agit, au contraire, de la représentation de cette exemplarité qui, chez Kant, se refuse à subsumer la réalité sous des lois

5 Une recherche sur Internet montre que cette citation continue à servir à la rhétorique politique américaine, de la gauche comme de la droite. Un exemple plus théorique est fourni par le professeur de droit, Cass Sunstein, qui l'utilise comme titre d'un de ses récents livres, A Republic, If You Can Keep It (Princeton, Princeton University Press, 2001).

$6 \quad$ Cette triade est soulignée souvent par Lefort. Cf., par exemple, L'Invention démocratique. Les limites de la domination totalitaire (Paris, Fayard, 1981), p. 118, et Essais sur le politique. XIX-XX siècle (Paris, Éd. du Seuil, 1986), p. 258. 
pré-établies et invite au jugement réfléchissant qui - bien que Kant n'en élabore pas la thèse - est le propre du jugement politique?.

La structure de cet article illustre un autre aspect de ce concept, car celui-ci ne propose pas simplement la résolution de problèmes préalables: bien compris, il en pose de nouveaux. On verra ici apparaître deux genres de questions. D'une part, comme il ne s'agit pas d'une synthèse mais d'illustrations exemplaires, les thèses avancées ne sont jamais univoques ni définitives. Elles se prêtent toujours à de nouvelles interprétations qui, à leur tour, éclaircissent le contexte d'où elles émergent. C'est pourquoi chaque partie se conclut par un examen de l'histoire repensée qui retrace la série de ré-interprétations historiennes des expériences examinées. D'autre part, le jugement politique qu'exprime l'histoire réfléchie s'ouvre à d'autres pratiques; l'histoire recommence, l'apparence d'une synthèse est déjouée et le réfléchi devient l'étoffe d'un nouveau moment du vécu. Ainsi, les trois grandes étapes de l'article peuvent être comprises, elles aussi, comme une sorte de triade qui répète à un niveau chronologique plus large la structure du jugement politique décrite au sein de chaque période plus réduite.

Le lecteur dira peut-être que je viens d'élaborer une théorie bien compliquée pour expliquer ce qui n'est qu'une orientation pragmatique et optimiste, typiquement américaine. Je réponds que l'histoire décrite ici est encore plus épaisse que la théorie. Cette histoire fonde et illustre une théorie qui ne prétend pas épuiser le sens de l'expérience. L'histoire comme sa théorie sont des interrogations qui se renouvellent constamment.

Le processus est parti du soudain changement introduit dans la vie des colons nord-américains par la victoire sur les Français en 1763 qui entraîna la réorganisation de l'empire britannique. Il leur fallut trouver une pensée politique critique permettant de comprendre cette situation tout en mettant en question la domination anglaise. Une fois élaborée, à la suite de maintes péripéties, cette théorie aboutit au besoin de déclarer l'indépendance en 1776. Mais celle-ci déclarée, il fut nécessaire de lui donner un contenu. Or ce qui venait d'être conçu au plan critique, tout en ayant rempli sa fonction initiale, se révélait inapte à assumer l'autonomie conquise. À nouveau s'imposait une double demande théorique, et une autre aventure commençait. Il était indispensable de réexaminer le vécu des ex-colonies depuis 1776 pour le doter de formes institutionnelles adéquates. La première conception de l'indépendance exprimée dans les constitutions des États et celle de la Confédération n'était pas capable d'encadrer la réalité sociale du pays libre. Remettre en route la réflexion s'imposait. Les uns vivront cette prise de conscience comme une crise presque débilitante alors que les autres sauront y saisir de nouvelles chances.Cette expérience réfléchie a fourni

\footnotetext{
Pour le détail philosophique de cet argument, je me permets de signaler mon livre, De Marx à Kant, (deuxième édition, traduction française, Paris, Presses Universitaires de France, 1995). Le rôle clé de la Critique de la faculté du jugement de Kant sur l'évolution de l'idéalisme allemand est souligné de manière lucide dans le monumental travail de Richard Kroner, Von Kant bis Hegel (Tübingen, J.G.B. Mohr -Paul Siebeck, 1921, 1924).
} 
le vécu qui fut à la racine des débats constitutionnels qui ont abouti à la (toujours actuelle) Constitution de 1787. Celle-ci a été conçue comme un cadre formel, rationnel et scientifique - on dirait aujourd'hui qu'elle est procédurale. Rien n'assurait sa mise en cauvre. Le débat sur la ratification fut difficile, passionnel et il en sortit de véritables clivages. La victoire fut incertaine. L'expérience accumulée depuis la lutte pour l'indépendance y a trouvé sa réflexion. Celle-ci dépasse en principe le formalisme de la procédure - mais il n'est pas sûr que les participants (et notamment les auteurs du Fédéraliste ${ }^{8}$ ) en avaient compris la nouveauté. On y voit de nouveau l'incertitude qui marque le moment réfléchi. L'histoire ne se termine pas sur une synthèse toute faite.

Le cadre d'interprétation proposé ici prend en compte l'incertitude propre au politique. La révolution américaine n'a pas trouvé son terme avec la ratification constitutionnelle, l'histoire s'est poursuivie, et la réussite n'était en rien garantie. C'est pourquoi cette étude devait poursuivre l'expérience pour prendre en compte dans un épilogue les conditions de possibilité de ce que les contemporains ont appelé "la révolution de 1800 ": cette élection vivement disputée qui vit le triomphe des partisans de Thomas Jefferson et le renvoi du présidant John Adams, qui pourtant se représentait.

En quoi s'agissait-il d'une révolution? Malgré la francophilie avouée des jeffersoniens, il ne s'agissait pas d'une révolution sociale complétant la transformation légale, comme celle des Jacobins en 1793. Nous y voyons plutôt la mise en cavre concrète de cette réflexion de l'histoire vécue depuis 1763 qu'exprimaient les débats sur la ratification de la constitution. La vision de la république sous le seul angle de la procédure est dépassée, ce qui permet l'expression de son complément démocratique. Cette nouvelle réflexion politique se manifeste et prend forme en deux temps. Le premier, c'est la passation paisible du pouvoir d'un parti politique à un autre - ce qui indiquait que l'on savait que la société était à la fois une et divisée, et que cette division (et plus largement la concurrence partisane) était nécessaire au maintien de son unité. Le second, en 1803, sur le même fondement social, c'est l'intervention de la Cour suprême pour invalider une décision votée par la majorité au pouvoir au nom d'une souveraineté et donc d'une unité supérieure à toute faction, minoritaire ou majoritaire, du peuple. C'est alors que, finalement, la réflexion aboutit et que la démocratie républicaine s'installe. Et, encore une fois, la structure qui se manifeste au travers de ces deux moments est celle qui caractérise le « jugement réfléchissant ».

J'ai dit au début de cet article que l'expérience américaine ne pouvait pas être transposée de toutes pièces dans n'importe quelle autre situation, et qu'elle n'était pas exempte de dérapages. Je ne décris pas ceux-ci, chacun citera ses propres exemples: l'histoire américaine ne vole pas de succès en succès. Il ne faut cependant pas dire n'importe quoi : la critique doit pouvoir s'expliquer mais la

8 La série d'articles de presse écrits sous le nom de Publius par Hamilton, Madison et Jay, et réunis plus tard sous ce titre pour défendre le texte constitutionnel en vue de sa ratification, sur lesquels nous reviendrons, naturellement, est un exemple parlant du jugement politique en mouvement. 
polémique n'y suffit pas, la morale encore moins. À l'opposé d'une histoire "exemplaire " qui articule les formes et les temps du jugement politique se situe ce que j'ai décrit ailleurs comme une «politique de la volonté ${ }^{9}$. Celle-ci advient lorsqu'un des moments constitutifs du jugement se trouve abusivement généralisé, séparé de son complément et traité comme autosuffisant. Dans un cas, la situation particulière qui appelait une intervention du politique est séparée de son contexte ; elle devient alors pour ainsi dire le mal en soi ; aucun débat n'est permis et tout compromis est exclu. Dans l'autre, le remède recherché est considéré comme universel, rien ne doit lui échapper, ce qui finit par l'instauration d'un mécanicisme formel et procédurier qui se veut englobant mais paradoxalement, exclut tout contenu réel. Ces deux possibilités structurelles décrivent le cadre dans lequel se situent les vrais dérapages, ceux que l'on ne dépasse ni par la polémique ni par la morale lesquelles ne sont, en fin de compte, que l'expression d'une volonté contrariée. Qui veut critiquer la démocratie républicaine américaine doit s'astreindre à ce cadre.

Afin de mieux mesurer la portée de l'histoire que l'on va lire, il est utile de revenir au contexte qui m'a amené à l'entreprendre. Ma préoccupation fut la critique, théorique mais aussi pratique, du totalitarisme. La vive opposition qu'inspirait alors ce genre de domination faisait naître l'espoir de voir naître une "révolution » anti-totalitaire ouvrant sur une société différente, où l'histoire reprendrait au-delà de l'opposition de deux systèmes dans lesquels l'économie occupait la place du politique. Les uns tablaient sur l'opposition de la société civile à un État centralisateur qui voulait étouffer une démocratie sociale ; les autres partaient d'une vision qui n'était guère éloignée pour retrouver ce qu'ils désignaient comme «le " politique. En France, on reconnaît d'une part la figure de ce qu'on appelait la " deuxième gauche » et d'autre part ce qui restait d'un espoir utopique qui jaillit en 1968 sur le fond d'un mépris parfois assez justifié des compromis « politiciens ».Il me semblait que l'expérience américaine aiderait à penser ces deux critiques du totalitarisme et leurs implications pratiques. Or, j'ai fini par comprendre que ces deux visions anti-totalitaires étaient trop univoques : l'une semblait supposer que la société pouvait exister sans l'État tandis que l'autre misait sur une vision du politique disjoint de la société réellement existante pour se donner corps et âme à un projet inouï littéralement "insensé ». Plus concrètement, les espoirs concentrés autour de la société civile furent écrasés entre autres par l'état de siège décrété en Pologne en 1981 ; ceux qu'avait éveillés le travail stimulant d'Hannah Arendt et son évocation des conseils ouvriers hongrois attendaient toujours le miracle. J'ai compris, pour ma part, que ces deux visions anti-totalitaires représentaient deux figures du dérapage du jugement politique. Il fallait aller plus loin et mettre en question la tendance que je désigne comme une politique de la volonté.

Cf. Dick Howard, Pour une critique du jugement politique. Comment repolitiser le jeu démocratique, (Paris, Éd. du Cerf, 1998). 
Il ne faut certes pas jeter le bébé avec l'eau du bain: les résultats de la critique du totalitarisme gardent leur actualité au-delà de la disparition de sa forme «réellement existante ». Il faut voir que le totalitarisme n'est qu'une expression d'un comportement plus général qu'on peut définir comme antipolitique. ${ }^{10}$ Il s'agit d'un refus de toute forme d'indétermination historique, et d'un rejet de toute division sociale, quelle que soit sa manifestation, refus et rejet qui se doublent de l'incapacité d'accepter la responsabilité de ses propres jugements. Nous avons là une des expressions d'une politique de la volonté, car une volonté ne peut pas, elle non plus, se permettre de telles épreuves.

C'est la critique de cette anti-politique que je poursuis ici. Cette tentation n'était pas étrangère à l'histoire américaine que je raconte, mais en fin de compte l'Amérique s'en est gardée, du moins pendant la période étudiée dans cet article. Par la suite, des dérapages eurent lieu : l'anti-politique réapparait, la tension de la conscience collective baisse, la vie civique se ramollit; à partir de là se crée un espace dans lequel peuvent s'engouffrer des craintes délirantes et des espoirs fous, une rapacité économique aussi bien qu'un nationalisme devenu d'autant plus agressif que l'on est moins sûr de soi-même. S'il ne faut pas s'étonner de voir se manifester de tels épisodes tristes et pénibles, il est surtout nécessaire d'insister sur le fait que ces excès ne sont ni nécessaires ni fatals.

Cet article n'entend pas pronostiquer une renaissance du politique aux ÉtatsUnis, il ne fournira pas davantage des recettes pour y parvenir : je prétends seulement décrire la structure qui permettra aussi bien d'identifier cette renaissance (quand elle apparaîtra) que de dénoncer les (trop fréquents) égarements antipolitiques. Depuis la chute du Mur de Berlin, on emploie le mot "démocratie " comme une sorte de passe-partout. Or, la distinction entre une politique du jugement et une politique de la volonté suggère une manière plus concrète et plus responsable de comprendre les possibilités mais aussi les égarements qu'ouvre la démocratisation de la vie sociale. Cette reformulation de ce qui oppose l'une à l'autre exprime aussi à sa manière le contraste entre la révolution américaine et sa cousine française, et fait voir au-delà de leurs différences la parenté des deux «exceptions » historiques.

On peut distinguer une république démocratique d'une démocratie républicaine. La première prend appui sur la volonté; elle part de la représentation d'une république, censée incarner la res publica, et se représente la démocratie comme

10 Peu après la chute du communisme, j'ai décrit ce que j'appelais les « deux cents ans d'erreur » qui suivirent les révolutions démocratiques en Amérique et en France. L'apparition de l'homme comme un être ayant des droits (des droits qui ne sont pas des " choses " ou une sorte de propriété, mais qui dépendent de relations entretenues avec d'autres hommes) créait la condition de possibilité de la politique moderne. Or, qu'il s'agisse du marché dit libre et fonctionnant à l'aide d'une « main invisible " ou de la vision d'un Plan réglant la société entière, d'une " juridification " des rapports sociaux ou encore d'un État Providence secourant ceux qui en auraient besoin, ce ne sont là que des cas de figure d'un rejet de la politique, et par-là du refus des acteurs politiques d'exercer leur jugement et d'en porter la responsabilité dans des situations où la décision tranche (et, comme dans le moment réfléchi, s'ouvre à une nouvelle expérience). Je poursuis cette intuition dans Pour une critique du jugement politique... Op.cit. 
un contenu ; le social vient ainsi au renfort du politique. Mais une société divisée où divers intérêts se font concurrence ne se laisse pas subsumer sous l'universalité de la république. Il faut alors que l'État intervienne pour transformer la société de manière à en faire un être homogène, transparent à lui-même, et en fin de compte uni comme l'est lui-même l'État. Il en résulterait une coïncidence de la société et de l'État, imposée par la volonté. Si jamais cette vision était réalisée, elle mettrait fin à l'histoire, et partant à la société elle-même, une fois pour toutes. On reconnaît ici la figure de la révolution française. À l'opposé de cette vision d'une république démocratique se situe celle d'une démocratie républicaine telle que l'a enfanté la jeune Amérique. S'étant trouvée indépendante, elle a dû faire face à la tentation d'un self government direct et démocratique à laquelle rien ne la préparait. Au fur et à mesure de son expérience - ce que va décrire cet article - elle a appris la nécessité de faire du "gouvernement du peuple par lui-même " une structure capable de s'auto-limiter, et cela passait par l'acceptation d'un cadre républicain. Dans ce mouvement, sont alors pris en compte les impératifs particuliers et pluriels de la dynamique sociale démocratique, tandis que celle-ci s'efforce de se donner une forme républicaine dont la législation valable pour tous reste toujours ouverte à la critique et à la révision. Cette rencontre des logiques démocratiques et républicaines n'est autre chose que la mise en cauvre du jugement politique. Reste maintenant à donner à cette figure un visage concret et une chair historique.

\section{La «révolution» de 1800}

La ratification populaire du travail de la Convention ne marquait pas la fin de la révolution, pas plus que les nouvelles institutions n'épuisaient les possibilités et les difficultés qu'aurait à affronter la jeune démocratie. Débattue dans des assemblées spéciales dont les débats exprimaient le caractère unique et solennel de cet acte constitutif, elle fut acquise de justesse, comme nous l'avons vu. Rien n'assurait la victoire de la cause nationale ni, non plus, la mise en cauvre réussie de la Constitution.

Le premier Congrès prit une décision de taille en proposant dix amendements (qui reprenaient des thèmes et des critiques évoqués par l'opposition lors du débat sur la ratification); ceux-ci constituent une sorte de Déclaration des droits. Mais cette dernière ne représente pas le fondement pour ainsi dire philosophique et politique de la constitution ; elle articule plutôt une série de corrélats procéduraux nécessaires à la bonne utilisation des nouvelles institutions libres ${ }^{11}$. Pour le reste,

\footnotetext{
11 Calquée sur le Bill of Rights anglais (1689), la Déclaration américaine vise surtout à protéger l'individu contre les actes d'un gouvernement arbitraire. Elle définit des garanties procédurales, ce qui fait que les droits qu'elle établit et protège sont, eux aussi, plutôt procéduraux que substantiels, ce qui fait que la démocratie républicaine américaine se distingue des constitutions fondées sur les prémisses sociales qui aboutissent à la création d'une république démocratique dont la révolution française fournit le modèle. Cf. Dieter Grimm, "German and American Constitutionalism. A Comparison», The Berlin Journal, n 7 , Fall, 2003, pp. 8-14
} 
on peut avoir l'impression que les débuts favorables des structures politiques imaginées par les conventionnels résultaient autant de l'inertie due aux conditions primitives de communication dans un pays peu peuplé et surtout préoccupé de son quotidien ${ }^{12}$ que du travail du Congrès lui-même. Ce dernier était dirigé d'une main de maître par Madison, auquel on doit le vote de la Déclaration des droits, alors qu'Alexander Hamilton, co-auteur du Fédéraliste, se chargeait de mettre en mouvement la branche exécutive à partir de son poste de secrétaire au Trésor. Tant que le vénéré George Washington présidait l'édifice, une harmonie régnait entre ceux qui, dira-t-on rétrospectivement, mettaient l'accent sur la qualité démocratique de la nouvelle Amérique, et ceux pour lesquels le renforcement de la république nationale et son avenir importaient davantage. Cette entente cordiale n'allait pas durer; la démocratie républicaine n'était pas encore établie, et la possibilité d'y parvenir demeurait fragile. Comme on le verra, c'est cette fragilité même qui fait de la vie politique américaine une création originale capable de se renouveler.

Comme souvent, les pressions extérieures firent apparaître les failles latentes du système. Si la scène domestique est restée plutôt calme durant les premières années de la nouvelle république indépendante ${ }^{13}$, la politique étrangère a brouillé les cartes et mis au jour des contradictions latentes, finissant par ouvrir des plaies qui mettraient longtemps à guérir. Il ne serait pas exagéré d'écrire l'histoire de la nouvelle république américaine comme une sorte de reflet du déroulement de la Révolution française - et surtout des péripéties de sa politique étrangère.

La première cause de division fut le dérapage conduisant à la Terreur. Jefferson, qui fut l'ambassadeur américain à Paris jusqu'à l'automne 1789 avant de rentrer pour prendre le poste de Secrétaire d'État dans le gouvernement de Washington, était francophile et démocrate passionné. Sa correspondance avec Madison foisonne d'éloges sur le déroulement de la Révolution (s'il évoque surtout l'action de ses amis Girondins, Jefferson ne condamnait pas la politique jacobine); elle donne aussi à penser qu'il souhaitait non seulement une alliance francoaméricaine pour s'opposer aux menées britanniques, mais peut-être même le renouvellement du mouvement révolutionnaire de $1776^{14}$. Cette francophilie des

12 Si la mise en oeuvre des nouvelles institutions frappe par sa routine, c'est par rapport à l'intense activité politique des années précédentes. Le travail des premiers Congrès et de l'exécutif est évalué avec force détail (et 900 pages) dans le livre de Stanley Elkins et Eric McKitrick, The Age of Federalism. The Early American Republic, 1788-1800 (New York, Oxford University Press, 1993).

13 Plutôt, et non pas tout à fait calme, car il fallut faire face à des mécontentements locaux qui, par moments, pouvaient prendre des dimensions nationales. Le plus connu fut la Whiskey Insurrection, qui éclata en 1794 lorsque des fermiers de l'ouest de la Pennsylvanie, contraints à bouillir leurs grains pour en faire du whisky, en raison des conditions primitives de transport, afin de pouvoir disposer de leurs récoltes, refusèrent de payer des taxes fédérales sur leur produit cru. La révolte fut de courte durée, la menace de fédéraliser les milices sous la direction de Washington calma les esprits - mais le fait de voir Alexander Hamilton prendre la direction des milices attisa les craintes de ceux qui redoutaient toujours une nationalisation de la vie démocratique locale.

14 Son collègue, Madison, ne se laissait jamais aller aux extrêmes devant lesquels Jefferson n'hésitait guère. Le livre déjà cité de Daniel Sissom, The American Revolution of 1800, relève un nombre surprenant de phrases qui ferait penser au lecteur non-avisé que Jefferson était un terroriste en 
partisans de Jefferson et de Madison ne fut pas sans retentir sur la politique domestique; elle les conduisit à considérer leurs différends avec la politique fiscale et manufacturière de Hamilton comme le point de départ d'une opposition plus fondamentale qui finit par donner naissance au premier système des partis politiques aux États-Unis. Ce résultat était pour le moins paradoxal en ce qu'il créait une situation considérée non seulement nuisible à l'unité de la jeune nation, mais dépassée par la révolution réussie et la ratification constitutionnelle. Or, contrairement aux craintes des contemporains, cette dynamique partisane allait devenir le moteur qui ferait prendre conscience aux Américains des contenus démocratiques insoupçonnés de leurs institutions procédurales. Mais il faudrait du temps avant que ce potentiel ne devienne visible et prenne corps dans la réalité, car le pays qui venait de conquérir son indépendance se concevait comme rassemblé par la recherche partagée d'un bien réellement commun. L'expérience montrerait que celui-ci existait, mais seulement au niveau symbolique, en tant que produit du conflit qui assure la dynamique de la démocratie. C'est alors que les thèses réfléchies dans Le Fédéraliste trouveront leur actualité.

La francophilie des partisans de Jefferson ne les poussait pas à s'opposer aux premières mesures intérieures du nouveau gouvernement, auquel participait d'ailleurs celui dont ils partageaient les idées. Sous l'impulsion de Hamilton, l'exécutif proposa et le Congrès vota la nationalisation des dettes contractées par les États pendant la guerre. Leur valeur, comme celle de la monnaie en papier et des assignats qui servaient à financer les institutions provisoires, était fortement dépréciée. Beaucoup de patriotes modestes les avaient vendues à vil prix à des spéculateurs pariant sur leur refinancement par le nouveau gouvernement. Hamilton en était conscient, mais sa proposition avait un double but politique qui lui semblait justifier l'opération. Elle attacherait les classes aisées à la cause de la nouvelle république dont la politique démocratique ne serait pas perçue (ainsi que l'enseignait la théorie classique) comme une menace contre la richesse. En même temps, cette politique raffermirait le crédit de l'État national, ce qui lui permettrait d'entreprendre une politique industrielle et le début d'un aménagement du territoire. À cette fin, une Banque nationale devait aussi être créée. Sur cette base, un second volet de la politique de Hamilton s'ensuivait : son fameux Rapport sur les manufactures proposait une politique tarifaire protégeant les jeunes industries américaines d'une compétition européenne qui viendrait s'établir au sein de la république grâce à des prix bas. Bien que cette politique désavantageât beaucoup

herbe, n'attendant que son moment. Or, s'appuyant sur les thèses de Bailyn, Sissom s'attache à montrer que la révolution dont Jefferson se faisait l'apôtre était non seulement démocratique, mais qu'elle devait être menée par des moyens plutôt pacifiques, comme ces comités de correspondance et « sociétés démocratiques » qui avaient montré leur utilité avant 1776 (Ibid., pp. 220 sqq.). Sissom a peut-être raison; mais il ne relève pas la contradiction qui voit Jefferson, démocrate décentralisateur, ne pas hésiter à soutenir la révolution jacobine centralisatrice. Et, comme le souligne John Patrick Diggins, John Adams, op. cit., lorsque Jefferson deviendra à son tour président, la politique sera tout à fait centralisatrice, qu'il s'agisse du maintien de la Banque nationale ou de l'achat de la Louisiane à Napoléon. Les thèses de Sissom, qui, pour être provocantes, en fin de compte, ne sont guère convaincantes. J'y reviendrai. 
de patriotes loyaux (qui faisaient entendre leurs voix), ces mesures se comprenaient comme un compromis soutenable. Sur le coup, personne ne songeait critiquer la tendance centralisatrice comme " jacobine », ni ne dénonçait les implications " aristocratiques » des choix de Hamilton (même si ceux qui avaient siégé à la Convention de Philadelphie se sont probablement souvenu de son intervention remarquée, au cours de laquelle il dit honnêtement, et en détail, ses préférences pour les institutions anglaises). Mais l'entente et le compromis n'étaient que temporaires, la politique étrangère allait se charger de faire apparaître une opposition de plus en plus définie.

C'est elle, en effet, qui allait dominer la vie de la jeune république ${ }^{15}$. Comme nous l'avons déjà vu, le Traité de Paris n'était toujours pas otalement entré en application - même si l'essentiel, la paix et l'indépendance était assuré - ni du côté américain, ni du côté anglais. D'âpres négociations commencèrent en 1793 sous la direction de John Jay ; elles aboutirent à un nouveau traité en 1794. Mais comme l'Angleterre était en guerre avec la France, et qu'elle refusait de reconnaitre la neutralité du commerce américain avec Paris, les futurs Jeffersoniens proposèrent de reprendre la tactique utilisée avec succès naguère, celle de l'embargo contre Londres ${ }^{16}$. Le traité de Jay ne garantissait pas la neutralité commerciale. En mars 1795, il fut rejeté par le Sénat qui revint sur sa décision en juin ; et la Chambre vota les fonds nécessaires à sa mise en œuvre en 1796. Cet épisode était significatif car ce qui n'était auparavant qu'une opposition entre " anglophiles aristocrates » et "francophiles républicains " au sein de l'assemblée débordait alors de ce cadre restreint. Cette distinction hautement symbolique allait maintenant colorer la vie politique entière. Ainsi, par exemple, les efforts parfois ridicules du vice-président John Adams pour donner à sa fonction des airs de dignité en singeant les mours parlementaires anglaises furent condamnés de façon si véhémente qu'on aurait pu croire que la république était en danger. Cependant, loin de ces détails formels, l'opposition partisane se cristallisait aussi sur des problèmes bien réels comme la question de savoir quelles mesures de défense nationale s'imposaient après la France eut réagi au traité de Jay par une politique que l'on qualifiait de « quasi-guerre ». La construction d'une force maritime et le remplacement des milices des États par une armée nationale ne pouvaient qu'éveiller les cauchemars qui hantaient la pensée vieux whig et restaient vifs chez les partisans de Jefferson. Le fossé entre les partisans d'une ligne et de l'autre se creusait.

${ }^{15}$ Ce qui démontre que l'Amérique n'était pas toujours aveugle devant le reste du monde, et que son réflexe premier n'est pas de se retrancher derrière une vision isolationniste, comme nous l'avons déjà suggéré plus haut.

${ }_{16}$ On se souvient que l'embargo avait contraint les Anglais à céder pendant les années 1760, l'intérêt bien compris des marchands anglais venant à l'appui des coloniaux. Mais cette alliance ne pouvait durer. Pourtant Jefferson et ses alliés continuaient à penser - pour des raisons qui restent obscures et tiennent peut-être à leur conception excessive de leur propre révolution, comme on le verra plus loin - qu'on pouvait brandir cette menace pour faire reculer les Anglais. Cette politique finit par provoquer une guerre en 1812 . 
La presse et les pamphlétaires dont on a vu l'efficacité entrèrent alors en jeu. La rhétorique, et souvent l'invective, échauffaient les esprits. Libre, la presse se partageait entre les deux camps, les plumes pouvaient s'acheter. L'administration de Washington (qui pouvait compter sur le soutien de la Gazette of the United States, dirigée par John Fenno et soutenu par des contrats d'imprimerie du gouvernement et des amis de Hamilton) n'en avait pas autant besoin que la toute fraîche opposition. Jefferson se servait de fonds provenant du budget du Département d'État pour aider au financement d'un journal, le National Gazette, dirigé par Philipe Freneau, employé comme traducteur au ministère.

Au-delà de la polémique, des questions de principe furent discutées par les ténors des deux camps. Hamilton publia déjà en 1793 une série de lettres intitulée Pacificus, à laquelle Madison, encouragé par Jefferson, répondit quelques mois plus tard sous le nom de Helvidius. Au coar de la controverse se situait le défi symbolique et le problème réel posés par la Révolution française. Mais sa manifestation concrète fut la compétition de plus en plus ouverte et rude des partis politiques naissants. Dans un pays neuf aux institutions inexpérimentées, cette rivalité était vécue comme une crise de la république alors que, rétrospectivement, on peut y voir le reflet d'une vie démocratique marquée par un débat contradictoire sans être fratricide. Mais avant que cette crise ne soit ressentie de façon dramatique, une épidémie de fièvre jaune poussa la classe politique à fuir Philadelphie. Puis la démission de Jefferson du Département d'État, à la fin 1793, contraignit Freneau à fermer la National Gazette (qui fut bientôt relayée par l'Aurora). Si l'harmonie n'était pas à l'horizon, la rupture ne l'était pas non plus.

Sans suivre en détail les aléas du débat, il faut souligner à quel point l'opinion le prit au sérieux. Deux " affaires " donnent la mesure des enjeux. Ce fut d'abord, en 1793, celle du "Citoyen Genêt », du nom du délégué de la Convention française auprès du gouvernement américain siégeant encore à Philadelphie (en attendant la construction de la capitale à Washington). Genêt délivrait à des capitaines américains des lettres de marque leur donnant le droit de capturer des vaisseaux de la marine anglaise. Non content de faire ainsi acte d'ingérence anti-anglaise, il encourageait également l'opposition naissante des amis de Jefferson à la politique de neutralité de l'administration Washington. Son influence fut telle que Hamilton publia une série de lettres sous le titre No Jacobin en vue de la contrer. Après un voyage triomphal à travers le pays, le gouvernement américain (par la voix de son secrétaire d'État, Thomas Jefferson lui-même, tenu par la solidarité gouvernementale à un moment où les divergences d'opinions n'avaient pas encore pris corps dans des partis organisés pour la bataille politique) demanda son rappel à Paris. Le Citoyen Genêt eut le bon sens de se marier avec une dame de la société philadelphienne plutôt que de rentrer vers un destin incertain.

La neutralité américaine continuait à poser des problèmes. Elle fut de nouveau mise en question après le départ du héros qui incarnait la vertu républicaine au-dessus des divergences d'opinion, George Washington dont le deuxième mandat se terminait en 1796 et qui choisit de retourner dans ses terres. L'Adresse des Adieux (Farewell Address) de Washington conjurait ses concitoyens 
de se tenir à distance des intrigues du Vieux Monde. Les élections placèrent John Adams en tête, devant Jefferson, ce qui fit, selon les dispositions légales qui ne prévoyaient pas l'existence de partis politiques, que le premier devint président et le second vice-président ${ }^{17}$. Adams n'eut alors pas le loisir de suivre le conseil de son prédécesseur. L'affaire connue sous le nom «XYZ " éclata en 1797. Son appellation suffit presque pour en expliquer la cause. Elle se réfère à trois émissaires du ministre des Affaires étrangères, Talleyrand, qui, après avoir contraint les ambassadeurs plénipotentiaires américains à faire antichambre pendant de longs mois, leur suggérèrent que le ministre pourrait bien discuter avec eux moyennant des sommes en argent liquide. Cette demande insultante fut ébruitée, ce qui accula les amis de Jefferson, connus pour leur francophilie, à la défensive. La meilleure défense étant souvent la contre-attaque vigoureuse, ceuxci firent appel au réflexe anticolonialiste, toujours vif, pour dénoncer l'anglophilie aristocratique des partisans du président. Aux différences politiques réelles s'ajoutait encore une fois la dimension symbolique qui renforçait et accentuait la division partisane naissante.

La présidence de John Adams mériterait à elle-même une étude. ${ }^{18}$ Nous connaissons déjà ce grand homme de la révolution, théoricien constitutionnel, pamphlétaire, diplomate et militant. Il incarnait une certaine vision républicaine sceptique de la Nouvelle Angleterre, face à la pensée démocratique qui émergeait, de façon inattendue, chez les cavaliers sudistes dirigés par Jefferson et encadrés par Madison ${ }^{19}$. Que faire de son vice-président? Ayant lui-même occupé ce poste, Adams aurait dû savoir qu'il serait difficile d'attendre de lui qu'il préside de

17 Il faudra amender la Constitution pour éviter de telles confusions. Le douzième amendement, ratifié en 1804, s'en charge. Mais cet amendement (qui sera le dernier voté avant ceux qui prennent en compte l'abolition de l'esclavage à la suite de la Guerre civile) conserve la disposition de 1787, qui oblige les Grands électeurs de voter pour au moins un candidat n'habitant pas leur État ; mais il ne mentionne pas l'existence de partis politiques. La division crainte par les pères de la nouvelle nation était toujours l'esprit de clocher. Le fait que les partis existaient bel et bien montre que, pas plus que les conventionnels, les Américains n'avaient toujours pas compris les implications de leur propre création. On se demande parfois s'ils la comprennent encore car la légitimité d'une opposition dans des moments difficiles ne va pas de soi, la surenchère est souvent tentante et la démagogie payante.

18 Le livre déjà cité de John Patrick Diggins, John Adams, op. cit. fait ressortir l'originalité de la pensée d'Adams à partir d'une critique suivie de Jefferson. Alors que l'étude de Diggins se concentre sur la présidence d'Adams, la grande biographie en deux volumes de Page Smith, John Adams (New York, Doubleday, 1962) donne une analyse biographique plus complète. La biographie plus populaire de David McCullough, John Adams (New York, Simon \& Schuster, 2001) est devenu un best-seller, ce qui réhabilite en quelque sorte un homme un peu oublié du grand public, plutôt jeffersonien, pour des raisons suggérées dans la belle étude de Merrill D. Peterson, The Jefferson Image in the American Mind (New York, Oxford University Press, 1960).

19 Madison se fait alors aider par James Monroe. Celui-ci deviendra président, après que Madison eut succédé à Jefferson, et prêtera son nom à la "Doctrine Monroe " (formulé et mise en oeuvre par son Secrétaire d'État, John Quincy Adams, le fils de John Adams). La "dynastie de Virginie " réussit à incarner une politique démocratique du fait de son opposition aux intérêts commerciaux du nord-est, quand bien même l'économie sudiste dépendait de l'esclavage. Cette apparente contradiction exprime le fait que l'opposition partisane naissante n'était que très partiellement fondée sur des intérêts matériels; ses racines étaient symboliques avant de trouver des ancrages réels. 
manière neutre le Sénat. En effet, Jefferson ne s'y plia pas; la scène internationale comme les conflits domestiques l'encouragèrent à affirmer son autonomie. La presse libre et les habitudes politiques du public y ajoutaient leurs effets. Les conflits domestiques étaient attisés par ceux qui opposaient la France et l'Angleterre. Celle-ci, acculée et menacée par la puissance française, insistait sur sa domination maritime; elle s'accordait le droit non seulement de violer la neutralité proclamée du commerce des États-Unis, mais encore celui d'inscrire sous son propre drapeau des marins américains faits prisonniers (pratique appelée empressment). L'honneur de la jeune nation était évidemment en jeu, les affaires aussi. La situation ne pouvait que rappeler celle que l'on avait connue avant la révolution, lorsque les Anglais cherchaient à imposer leur système mercantile. La réplique qui venait à l'esprit était encore la même : l'embargo. Proposé par les alliés de Jefferson, c'était un remède qui, comme par hasard, coïncidait parfaitement avec la politique française de l'époque! On ne sera pas surpris d'apprendre que l'administration Adams préférait la négociation... et qu'elle était soutenue par les intérêts commerciaux de la Nouvelle-Angleterre, alors que ceux de l'agriculture sudiste pensaient tout à fait pouvoir survivre et même prospérer en raison des effets d'un tel embargo ${ }^{20}$.

Le climat politique était de plus en plus tendu, la presse se déchaînait. Le Congrès et les partisans d'Adams finirent par intervenir, ce qui aboutit, en 1798, au vote de quatre propositions connues sous le nom Alien and Sedition Acts. Elles préconisaient des mesures apparemment radicales, et rencontrèrent une réponse tout aussi radicale des partisans de Jefferson. S'agissant des " aliens", la loi devait viser d'abord Albert Gallatin, un Suisse qui avait remplacé Madison à la tête des républicains au Congrès (et qui sera Secrétaire au Trésor sous la présidence de Jefferson); mais sa portée pouvait disqualifier et à renvoyer tout immigrant un peu trop remuant. Par-là, elle rejoignait le texte qui voulait interdire la " sédition " terme dont le contenu était difficile à définir. Le but était évidemment de contrôler une presse devenue trop virulente, taxée d'irresponsabilité, et - ce qui ne pouvait manquer de raviver les dénonciations de l'aristocratisme des partisans d'Adams accusée de manquer de respect pour les institutions. Sévères, ces lois pourtant avaient une grande faiblesse : elles étaient difficilement applicables et, en fin de compte, elles ne le furent presque pas. Ce qui, évidemment, contribua à leur discrédit mais aussi à celui des hommes politiques qui les avait fait adopter. Il y avait un effet encore plus grave, car ces lois furent votées par les représentants d'une démocratie dont pourtant elles menaçaient les bases mêmes ${ }^{21}$. Sans le vouloir, leurs auteurs posaient ainsi un problème qui n'était pas seulement conjoncturel.

20 On s'étonne à l'idée que les Américains pensaient pouvoir tenir tête à la puissante économie anglaise, même si elle était affaiblie par les années de conflit avec la France. Pour les comprendre, il faut insister à nouveau sur l'idéalisme universaliste qui sous-entendait leur révolution. C'est la thèse, déjà évoquée, de Felix Gilbert, To the Farewell Address, op. cit.

${ }^{21}$ Comme je l'ai suggéré plus haut, il faudrait distinguer entre les procédures démocratiques et ce qu'on pourrait désigner comme le vécu démocratique. 
La réponse ne tarda pas à venir. Elle était mise en caure de manière assez subtile par Jefferson et surtout par Madison ${ }^{22}$. L'un, s'occupant de la Virginie, l'autre du nouvel État du Kentucky, firent voter des Résolutions visant à annuler ces lois qu'ils décident de ne pas mettre en œuvre chez eux. Venant de Jefferson, la thèse pouvait se comprendre : il s'agissait de protéger la liberté menacée par un pouvoir centralisateur ; les droits de l'homme précédaient et conditionnaient, à ses yeux, toute politique. La vision des fédéralistes alliés d'Adams privilégiait l'unité nationale au détriment de cette liberté pour laquelle les patriotes avaient combattu, tandis que Jefferson la mettait au-dessus de tout (y compris parfois, il faut le dire, la cohérence de sa propre théorie ${ }^{23}$ ). Le fait que Madison l'ait soutenu (dirigeant même en sous-main l'opération) pose problème, car il s'agissait, en dernière instance, de réclamer l'autonomie des États formant la république, ce qui constituait justement, selon Madison lui-même, le fond des "vices " de la Confédération tels qu'il les avait dénoncés avant de partir pour Philadelphie ! Son "Plan de Virginie ", qui avait orienté les débats constitutionnels, insistait sur la nécessité d'un veto national sur les lois des États (pour éviter l'anarchie dénoncée dans la démocratique Pennsylvanie). Comment et pourquoi défendait-il maintenant une politique contraire à cette première inspiration? On peut arguer que dans des contextes différents, les mêmes principes se défendaient d'une autre manière, selon les enjeux et les rapports de force. Créditons donc Madison (et même Jefferson, encore que le cas soit plus compliqué) d'une vraie constance philosophique et regardons de plus près la situation.

La révolution était encore dans toutes les mémoires; ses représentations idéologiques aussi. On ne voulait pas reconnaître l'existence de divisions au sein de la république alors même que l'opposition symbolique des deux parties était présente à tous les esprits, mais on pouvait dénoncer l'anglophilie et l'aristocratisme du gouvernement Adams. Selon le schéma vieux whig, il fut donc accusé de vouloir étendre son pouvoir aux dépens de la bonne foi du peuple innocent. La preuve définitive en était fournie par les Alien and Sedition Acts. Les Résolutions du Virginie et du Kentucky répétaient le geste de 1776 ; il fallait se

${ }^{22}$ Cf. Daniel Sissom, The Revolution of 1800, op. cit., qui suit dans le détail, à travers la correspondance des deux comparses avec leurs collaborateurs, ce qu'il ne veut pourtant pas désigner comme un complot, bien qu'il relève les consignes données par exemple de brûler telle lettre, de taire telles intrigues ou d'entreprendre en douce telles actions. Sissom, on l'a vu, préfère y voir un retour aux méthodes "révolutionnaires " déjà pratiquées en 1776. Sa thèse, me semble-t-il, est à cet égard anachronique, comme sa vision d'une " révolution ».

23 Passons sur la question de l'esclavage, qu'on ne pourra pas résoudre ici. Il faut tout de même se poser la question du "révolutionnarisme " de Jefferson. Sissom, comme d'autres auteurs, cite maintes phrases où Jefferson dit, par exemple, préférer une presse libre à un état stable, où il affirme - à la manière de Thomas Paine - qu'il faut tout simplement revenir aux conditions naturelles et pour ainsi dire d'avant la civilisation, pour jouir en paix d'un bonheur qu'on n'aurait plus à " poursuivre ». Mais celui qui parle ainsi, comme le note John Patrick Diggins, John Adams, op. cit., est un esclavagiste, toujours endetté, incapable de survivre des fruits de son propre travail. Chacun pourra faire appel à "son " Jefferson, telle Hannah Arendt qui, dans son Essai sur la révolution veut voir dans sa proposition d'élections locales (le ward system) un lointain ancêtre de l'idée des conseils ouvriers de la révolution hongroise de 1956. 
séparer de la racine du mal avant qu'il ne soit trop tard. Mais les circonstances n'étaient plus les mêmes. Et le sombre et expérimenté puritain qu'était Adams n'allait pas se laisser faire ; il connaissait et la chanson et la répartition des rôles. Or, les acteurs n'étaient pas eux non plus les mêmes; derrière l'opposition partisane symbolique se trouvaient d'autres intérêts et des rivalités qu'on avait du mal à identifier mais dont l'existence était connue. L'ambiguïté des hommes et l'incertitude du paysage politique était indiquée par le fait que Hamilton, qui avait quitté le gouvernement, dirigeait une opposition qui avait déjà réussi à manipuler des membres du gouvernement d'Adams. Mais tout en étant toujours critique à l'égard de la francophilie jeffersonienne, Hamilton se trouvait aussi aux prises dans la politique new-yorkaise avec celui qui était considéré par l'opinion publique comme le colistier de Jefferson, Aaron Burr (même s'il n'y avait toujours pas d'appareils politiques constitués) . La bataille électorale de 1800 fut amère, ses enjeux apparemment simples n'étaient pas si clairs. Bien qu'Adams fut finalement battu, l'élection ne pouvait pas être réglée entre Grands Électeurs car Jefferson et Burr étaient arrivés à égalité. Il fallut s'en remettre au Congrès. C'est in extremis que Jefferson sortit vainqueur, après trente-six tours de scrutin (et avec le soutien inavoué de Hamilton ${ }^{24}$ ). Malgré ces complications, ses partisans se mirent à parler de la "révolution de 1800 ". $^{25}$. On les comprend sur le plan symbolique, mais le concept de révolution prête à confusion.

Dans son célèbre Discours inaugural, le 4 mars 1801, Jefferson fait allusion aux amers conflits que la jeune démocratie républicaine venait de vivre et appelle à la réconciliation. Reconnaissant la division partisane, Jefferson insiste pourtant sur l'unité nationale: "Nous sommes tous des Républicains, nous sommes tous des Fédéralistes. " Mais, poursuit-il en substance, il existe des hommes honnêtes qui mettent en question la force d'un gouvernement républicain. L'existence de tels hommes fournit la meilleure preuve de la puissance du gouvernement libre de l'Amérique. Mais la définition que donne Jefferson des obligations du pouvoir est minimale. Il s'agit " d'empêcher les gens de faire mal les uns aux autres, [et] de les laisser libres pour régler leur propre poursuite industrieuse d'améliorations, et de ne pas prendre de la bouche du travailleur le pain qu'il a gagnér6 ". Si révolution il y a, elle n'est donc certainement pas jacobine! L'unité nationale qu'elle veut alors rétablir ne s'impose pas. Le nouveau gouvernement ne se propose pas d'intervenir dans le vécu des gens; comme l'affirmait déjà la Déclaration d'indépendance, le bonheur est toujours à "poursuivre ", ce qui est

${ }^{24}$ Ce qu'envisageait Hamilton n'est toujours pas connu avec certitude. Il trouva la mort, en 1803, dans un duel (pour des raisons non politiques) avec le même Aaron Burr. Ce dernier, vice-président de Jefferson, finira disgracié au terme d'une aventure politico-spéculative, sur la frontière occidentale.

25 Ils s'identifiaient souvent comme des " démocrates-républicains », bien qu'ils ne donnaient pas à ce concept le sens que nous lui prêtons dans ce article. Il s'agissait simplement de s'identifier comme des amis du peuple à l'opposé des partisans d'un gouvernement national, qui pour leur part conservèrent le label de fédéralistes (et qui furent dénoncés comme " aristocrates »).

26 Jefferson, Writings, op. cit., pp. 493, 494 
une autre manifestation de la démocratie. Il est vrai que la pratique de Jefferson ne coïncide pas toujours avec sa théorie, comme on l'a vu à propos des résolutions de la Virginie et du Kentucky. Le gouvernement modeste qu'annonce ce Discours inaugural devra faire face aux réalités de la politique domestique et surtout internationale.

Mais ces contradictions, et les bonnes intentions de Jefferson sont moins importantes qu'un autre aspect de cette "révolution de 1800 ». Malgré la défaite d'Adams, les Fédéralistes n'avaient aucune raison de se donner comme battus ${ }^{27}$, or cette élection marque leur disparition comme acteur politique. En fait, on atteignait, provisoirement, le point culminant du processus politique commencé en 1763, et c'était là que se situait sans doute la vraie révolution. L'évincement des Fédéralistes est en effet lui-même moins surprenant que le paisible transfert du pouvoir qui fit suite à une campagne politique agitée où les uns et les autres agitaient l'épouvantail révolutionnaire. Les Résolutions de la Virginie et du Kentucky exprimaient de leur côté la menace d'une dissolution de la nation, alors que les Alien and Sedition Acts étaient utilisés contre les opposants du gouvernement. Malgré ce qu'on avait pu regarder comme un prélude à une guerre civile, pour la première fois dans l'histoire politique mondiale, le pouvoir passait tranquillement d'un parti politique à un autre, personne n'était contraint à l'exil, jeté en prison ou promis à la disgrâce sociale ou civile. Comment comprendre cette innovation? Il ne s'agissait pas d'un renversement de majorité parlementaire mais d'un mouvement de société. Si le conflit partisan représente la forme conçue de l'expérience de l'indépendance, cette élection en fournit la forme réfléchie, le moment où cette expérience originaire de la vie politique américaine réalise ce qu'elle portait comme potentiel politique. Mais les protagonistes ne comprenaient pas ce qu'ils étaient en train de vivre. Ils ne se rendaient pas compte de la leçon à tirer de leur propre histoire politique, à savoir que la représentation politique de la souveraineté populaire est symbolique. La défaite des Fédéralistes s'explique par le fait que dès lors que cette souveraineté symbolique se prend pour objective elle se trahit en se présentant comme ce qu'elle n'est pas, ce qui met en danger la liberté concrète du peuple souverain - comme ce fut le cas lors du vote (pourtant légitime du point de vue de la procédure) des Alien and Sedition Acts. Inversement, c'est le vécu de cette structure symbolique du politique qui explique la radicale innovation que les contemporains concevaient selon les catégories de la "révolution ». Tout comme le gouvernement établi par la constitution de 1787,

Il ne s'agit pas ici de reprendre l'argument du livre de Garry Wills, The Negro President, op. cit., qui insiste sur le fait que l'élection de Jefferson n'était pas l'expression de la volonté populaire mais plutôt l'effet du pouvoir esclavagiste (résultant du poids des esclaves qui comptaient pour troiscinquième d'une personne dans le recensement). Ce qu'il faut comprendre, c'est que les Fédéralistes ne disparurent pas du jour au lendemain, ni non plus que l'unité réelle du pays ne fut rétablie d'un seul coup. Un autre processus était en train de prendre corps, le terrain du politique se transformait. Si les hommes dont les opinions et les intérêts avaient été exprimés par la politique des Fédéralistes ont bel et bien continué à vivre leurs vies, l'opposition symbolique qui façonnait leur existence en tant que force politique fut mise hors jeu. 
dont Le Fédéraliste explique la légitimité, le peuple souverain peut (et doit) être à la fois un et divisé. Ce n'est qu'à cette condition, paradoxale à première vue, qu'une démocratie perdure ${ }^{28}$.

La confirmation de la leçon vécue lors de l'élection de 1800 eut lieu trois ans plus tard. Dans l'intervalle de trois mois qui séparait l'élection de Jefferson de sa prise de fonctions, le parti de John Adams, toujours au gouvernement, prit quelques "précautions ». Des fonctionnaires et magistrats furent désignés, les derniers étant nommés, disait-on, le dernier soir, à minuit, juste avant l'installation de Jefferson à la présidence. On comprend que la nouvelle majorité ait mal pris mal cette affaire; Madison, le secrétaire d'État, refusa d'accepter la nomination d'un magistrat dénommé Marbury qui, pour sa part, porta plainte devant la Cour suprême (présidée par John Marshall, lui-même désigné par Adams après sa défaite). Par une décision assez compliquée, tout en déboutant Marbury de sa demande au motif qu'elle était mal fondée légalement, la Cour ne donnait pas raison à Jefferson et ses $a^{2}$ s. $^{29}$. Sans entrer dans le détail de l'argument jurisprudentiel, ce qui frappe c'est que le résultat du procès Marbury v. Madison réitérait la structure réfléchie de l'expérience politique de 1800. La Cour, qui n'avait eu jusque là qu'à statuer sur des arbitrages secondaires, ne pouvait pas se tenir en dehors des conflits politiques qui devenaient de plus en plus aigus. Il semblait que les amis d'Adams y trouvaient un dernier refuge, et une manière de préparer leur revanche. John Marshall ne pouvait donc en aucun cas justifier son arrêté par un argument partisan. Cette contrainte explique la portée plus générale qu'acquit sa décision, qui insistait sur le fait que la Cour devait être en dernier lieu le garant des institutions forgées en 1787, dont elle était elle-même issue. Il s'ensuit que la majorité, par essence temporaire, aux commandes du gouvernement ne peut prétendre incarner le peuple souverain; celui-ci ne s'incorpore pas plus dans le législatif que dans aucune autre branche du gouvernement. Sa représentation est encore une fois symbolique; elle se réfléchit dans la constitution, qui transcende toute majorité et toute institution. Cette constitution, conçue par ces auteurs comme procédurale acquiert alors une toute autre épaisseur. Elle n'est plus simplement la garantie de la forme républicaine des institutions; on voit maintenant comment la république non seulement permet et encourage mais, en dernière instance, dépend elle-même des conflits et de la pluralité toujours réaffirmée de la vie démocratique. Par-là se manifeste un autre aspect de la démocratie, celui qui fait qu'elle peut déraper lorsqu'elle cède à la

${ }^{28}$ C'est la grande erreur du travail de Daniel Sissom, qui veut dépasser la division qui constitue selon lui la négation de la vraie démocratie. S'il accepte, par moment, l'idée d'un conflit de principes, il refuse la politique partisane. Son idéal, qu'il prête à Jefferson, serait un État sans partis, ou au mieux monopartite. Cf. The Revolution of 1800, op. cit., p. 444.

29 On trouvera une explication concise du fondement jurisprudentiel de cette décision dans Forrest McDonald, States' Rights and the Union, op. cit., pp. 52sqq. McDonald note aussi que les partisans de Jefferson n'acceptaient pas sans protestation la légitimité du pouvoir judiciaire. Ils essayaient de prendre leur revanche en réclamant l'impeachment d'un des juges fédéralistes - et il ne furent pas loin de gagner! On voit par là, encore une fois, que la réussite américaine n'était pas prédestinée, l'échec était toujours possible. 
tentation de se prendre pour objective. C'était l'erreur des Républicains jeffersoniens qui avaient refusé la nomination de Marbury. Plus généralement, cette erreur résulte de la volonté de dépasser le conflit, de raboter les inégalités et les adversités, et d'incarner l'unité populaire. Cette tentation volontariste n'est pas facile à éviter.

Ce problème se manifeste alors que les Républicains vont dominer la vie politique durant les vingt-quatre années qui vont suivre. La guerre avec l'Angleterre finit par éclater en 1812. En réaction, un dernier sursaut des Fédéralistes fut d'appeler à la réunion d'une "Convention » à Hartford qui (à l'instar des Résolutions de Virginie et de Kentucky) devait considérer la sécession. Mais la guerre (qui vit la capitale, Washington, en flammes) prit fin assez vite. Au lendemain de son élection en 1816, James Monroe proclame "l'ère des bons sentiments », censée consacrer, la réconciliation. Mais l'unité retrouvée ne dura pas longtemps, le conflit refit surface. L'élection de 1824 fut chaudement contestée, et comme pour celle de 1800 le Congrès dut arbitrer. Son choix de John Quincy Adams fut critiqué comme le fruit d'un arrangement corrompu (qui donnait à l'un des candidats, Henry Clay, le poste de secrétaire d'État). La contestation était vive, les grandes ambitions nationales d'Adams furent déçues alors qu'un nouveau système de partis commençait à prendre racine. Sous la houlette d'Andrew Jackson, une tendance qui donnerait naissance au Parti démocrate prépara puis remporta les élections de 1828, se targuant d'être la voix du peuple prenant sa revanche sur le régime de la corruption. Il n'est pas nécessaire de souligner qu'elle ne sera pas plus capable qu'une autre de se maintenir au pouvoir sans devoir affronter la contestation (qui viendra cette fois du Parti whig), puis rencontrer la défaite. Elle laissait pourtant sa marque symbolique, car le vice-président et successeur de Jackson, Martin Van Buren, fut le premier homme à faire de sa carrière d'homme de parti le marche-pied pour accéder au poste suprême de la république.

\section{Conclusion}

Les acquis de la réflexion de la révolution de 1800 sont restés fragiles. La difficile légitimation de la politique partisane dépendait de l'acceptation du conflit dans une société dont le pluralisme nourrissait l'énergie démocratique. Pareillement, l'intervention de la Cour Suprême pour imposer à la démocratie des limites qu'elle ne savait pas se fixer elle-même, rencontrait, elle aussi, des obstacles inhérents à son fonctionnement même. Si la Cour a réussi à s'imposer dans Marbury v. Madison, il faudra attendre encore cinquante ans avant d'intervenir à nouveau pour invalider une loi votée par le Congrès! Il est vrai qu'elle proposera des arrêts de dimension nationale en invalidant des lois adoptées par des États, mais ce faisant, elle réalisera seulement, d'une certaine manière, le vœu de Madison présenté dans le Plan de Virginie. Une telle interposition juridique 
n'a pas, en général, de portée politique directe ${ }^{30}$. Ce n'est qu'au moment où elle en acquiert que se posent des problèmes (et quand cela adviendra, ils seront réglés par une guerre civile!). À ce moment-là, en effet, la valeur symbolique de l'action constitutionnelle déchoit, sa réalité se fait sentir comme illicite parce que partisane, le jugement rendu est perçu comme biaisé et résultant, littéralement, d'un pré-jugé. Prise entre un attachement formaliste à la procédure et la nécessité de rendre une décision qui prenne en compte le pluralisme conflictuel d'une démocratie, la Cour doit trouver son chemin sans se laisser aller à pencher trop d'un côté ou de l'autre. Elle n'y parvient pas toujours. C'est dire qu'à l'évidence, ici aussi, le vécu, puis le conçu appellent le troisième temps, celui de la réflexion.

Naturellement, l'histoire américaine ne s'achève pas avec " la révolution de 1800 ", mais pour ce qui nous occupe - tirer les leçons de ses origines - il n'est pas nécessaire de poursuivre l'examen au-delà de ce qui nous est apparu à la fois comme l'épilogue de la naissance d'une démocratie républicaine et comme l'expression de la réflexion politique, Celle-ci se manifeste dans la passation paisible du pouvoir d'un parti à l'autre en même temps que se met en place une jurisprudence qui affirme la priorité du pouvoir constitutionnel comme représentation symbolique de la souveraineté populaire. La coexistence des partis reflète la pluralité et la division qui sont constitutives de la vie démocratique, alors que l'efficacité de l'intervention de la Cour exprime le cadre républicain au sein duquel peut, et doit, fonctionner cette démocratie. À regarder de plus près la mise en cavre de cette double structuration de la démocratie républicaine, on voit que le rôle des appareil politiques est de porter à l'attention du public les questions, les intérêts et les souhaits particuliers de l'un ou l'autre élément de la société. Au mieux, l'un saura intégrer ces priorités et réussira à les faire accepter par le gouvernement en place. L'autre (pour rester dans le schéma bipartite) cherchera à organiser ses propres partisans pour proposer sa vision. À supposer que l'un ou l'autre camp prédomine pendant un laps de temps, il y a toujours le danger qu'il pousse trop loin sa domination, imposant sa volonté sur la scène politique. À ce moment-là, l'instance judiciaire pourra éventuellement s'interposer, car l'une de ses fonctions (qui n'est pas simplement procédurale) est d'assurer la conservation, voire la protection, du terrain du politique, à savoir les règles du jeu républicain. Mais une telle intervention n'est pas infaillible, son formalisme légaliste court, pour sa part, le risque de paralyser la vie politique partisane, qui devra se mobiliser contre une "république des juges ». En d'autres termes, on voit une complémentarité entre la démocratie partisane et le cadre républicain. Une telle coexistence positive n'est pas instituée une fois pour toutes, elle est même assez rare, mais son existence marque des moments où la vie politique vaut d'être vécue.

\footnotetext{
Il faut qualifier cette affirmation. Les interventions de la Cour avaient bien sûr des conséquences politiques, mais elles étaient le résultat de décisions de portée avant tout économiques. Cf. Morton J.Horwitz, The Transformation of American Law, 1780-1860 (Cambridge, Harvard University Press, 1977).
} 
Hélas, la précarité de cette structure réfléchie est évidente, et l'on ne peut pas prétendre qu'il suffit d'une volonté généreuse pour ré-allumer des feux souvent éteints. Mais il est peut-être concevable de faire de cette impossibilité volontariste une vertu, d'autant plus que l'un des dangers de la vie en démocratie tient, nous l'avons vu, dans son incapacité à se limiter, dans sa difficulté de saisir et d'opérer la distinction entre son fondement symbolique et sa réalité concrète. C'est que la démocratie a du mal à comprendre et à mettre en oeuvre le jugement politique. Elle tend à devenir un volontarisme qui s'imagine comme l'incarnation du peuple souverain : après tout, ce peuple est un, son unité s'exprime par une volonté unie alors que la division conduit à l'hésitation sinon à la paralysie. Au contraire d'un volontarisme binaire, marqué au coin du tout ou rien, le jugement politique réaffirme les conditions toujours singulières et la multiplicité rebelle à l'unification immédiate. Ce jugement, comme la prudence, ne cherche pas à subsumer ses éléments constitutifs sous un universalisme (ou un moralisme) dont la généralité ne laisse plus de place à la responsabilité individuelle. Il insiste, évidemment, sur la vérité, et donc sur la validité générale de ses constations; mais celles-ci se vérifient dans la confrontation avec les propositions avancées, également dans l'ordre du jugement, par les autres participants à la vie politique. Pour cette raison, il est toujours révisable et ne peut jamais exclure les opinions des autres. Pour que la démocratie perdure, il faut qu'elle sache se limiter, ce qui demande l'apprentissage du jugement politique.

Mais une chose est d'affirmer son rôle au sein d'une démocratie établie, une autre est d'en montrer l'origine. C'est justement à cela que sert la complémentarité structurelle entre la politique partisane et le contrôle de la constitutionalité exercé par la Cour suprême. En effet, le jugement politique comporte deux moments. Il faut d'abord identifier un problème singulier, une situation qui ne peut pas être réglée de manière satisfaisante dans le cadre des lois existantes. Il faut ensuite proposer une législation qui la prenne en compte sans en nier la particularité. L'expérience américaine illustre deux manières d'y parvenir. Dans le premier cas de figure, la concurrence partisane démocratique fait ressortir les instances particulières qui demandent à être confrontées ; les positions sont ensuite débattues au niveau politique afin d'élaborer, pour elles, des propositions de loi valables universellement; mais comme celles-ci s'appliquent toujours à des cas spécifiques, elles devront éventuellement subir l'épreuve de l'examen de leur validité constitutionnelle à partir de nouvelles conditions particulières. C'est ici que se situe la seconde possibilité. Il arrive que la vie politique soit bloquée, les partis ne pouvant pas se décider à traiter d'une question brûlante, tandis que les citoyens démobilisés ou pris dans leurs affaires privées détournent l'attention. Si le problème prend une tournure plus pressante, il faudra que la Cour intervienne pour le faire apparaître sur la scène publique. À partir de là, les partis ne pourront plus l'ignorer, ils devront s'en saisir pour formuler une législation. Ce faisant, ils délesteront la Cour d'une fonction politique qui n'est pas la sienne. D'une éventualité à l'autre, la structure reste la même, mais les acteurs ont changé de rôle : dans le premier cas, les partis - c'est-à-dire le champ démocratique - identifient le 
problème particulier et, à partir de sa mise en forme législative, la Cour lui accorde une légitimité républicaine; dans le second, la Cour pointe la difficulté, pour la faire entrer dans le champ de la res publica, et la lutte partisane se charge de lui fournir une légitimité démocratique.

L'argument peut paraitre abstrait. Il serait possible d'illustrer ces cas de figure à travers l'histoire américaine (surtout en ce qui concerne la catégorie, élastique et donc de plus en plus vaste, des droits civiques), mais le sceptique n'aurait pas de mal à trouver des contre-exemples. Et, faut-il l'avouer? le sceptique aurait de quoi avoir raison. J'ai déjà insisté là-dessus : rien ne prédestinait l'expérience américaine à réussir, et ses institutions ne peuvent pas être exportées urbi et orbi. Ce qu'on peut tirer de cette histoire des origines de la démocratie américaine se réduit, dans une certaine mesure, à cette figure du jugement politique que je viens d'exposer. Elle ne tient pas dans le jugement d'une personne, et elle n'est qu'indirectement le jugement du peuple souverain. Sa condition de possibilité est illustrée par l'histoire vécue, conçue et puis réfléchie de la jeune Amérique. Ce que nous apprend cette histoire, ce n'est pas la manière de construire des institutions qui pourrait y conduire. Poser la question ainsi, ou plutôt entrer dans un tel projet que l'on pourrait qualifier de state building - les exemples ne manquent aujourd'hui en cette matière - serait une nouvelle manière renouer avec le volontarisme politique. Ce que nous pouvons apprendre de l'expérience américaine, c'est d'une part à reconnaître de telles instances d'une politique du jugement lorsqu'elles apparaissent, et d'autre part à éviter la dérive trop fréquente qui consiste à remplacer le jugement politique par une politique de la volonté. Enfin, reconnaitre la politique du jugement dans l'histoire que nous venons de parcourir nous fait comprendre pourquoi les dérapages (fréquents) font partie des risques inhérents à tout exercice démocratique. Il est trop tôt pour évaluer les résultats des élections de 2004 que j'ai évoquées au début de ces considérations historico-philosophiques. Une phénoménologie historique ne se laisse pas contraindre par une dialectique restée linéaire et pan-logique malgré elle. ${ }^{31}$

Le passage qui conduit de l'analyse génétique à la critique normative avant de revenir à cette première orientation rend possible et même nécessaire une philosophie du jugement. La forme que pourrait prendre à son tour une politique du jugement adéquate à notre monde devenu interdépendant tout en maintenant une vision politique "Westphalien " reste à imaginer. Ce qui est certain, c'est que les ambiguités pour ne pas dire les antinomies de la démocratie resteront un défi qu'on néglierait à ses risques et périls.

31 Je fais allusion à la lecture sympathique mais finalement critique de Marx que je propose dans la dernière partie de The Specter of Democracy, op. cit.. Une première version de cette lecture critique fut publié dans Veritas, Vol 49, Nr. 1, Março 2004. 